\title{
THE BLIND DATE: THE EFFECTS OF PASSENGER CONVERSATION AND GENDER ON LOOKED-BUT-FAILED-TO-SEE (LBFTS) ERRORS
}

\author{
Cale White ${ }^{1} \&$ Jeff Caird ${ }^{2}$ \\ ${ }^{1} \mathrm{AECOM}$ \\ ${ }^{2}$ University of Calgary \\ Calgary, Alberta, Canada \\ Email: Cale.White@aecom.com, jkcaird@ucalgary.ca
}

\begin{abstract}
Summary: This study examined situations where drivers looked but failed to see hazards (LBFTS), and whether passenger conversation and gender affected hazard detection rates. To reliably produce LBFTS errors, 40 young drivers $(M=20.3)$ encountered motorcycles and pedestrians while making left turns in the University of Calgary Driving Simulator (UCDS). Prior to turn initiation the UCDS screens flickered using an extension of change blindness methods. In addition, drivers either drove alone or conversed with an attractive confederate passenger. Measures of LBFTS errors, hazard detection and social factors were analyzed. Higher rates of LBFTS errors and hazard detection occurred while conversing than while driving alone. A discriminant function analysis (DFA) using conversation and gender as predictors accurately classified LBFTS errors. Higher passenger attraction and higher extroversion were related to missing more critical events. The basis of LBFTS errors in divided and selective attention and classification implications are discussed.
\end{abstract}

\section{INTRODUCTION}

'Looked-but-failed-to-see' (LBFTS) driving errors, where collisions involve a driver who fails to detect another roadway entity despite looking directly at it, was coined in the 1970's (Hills, 1980; Treat, 1980). Over the last four decades crash data and case studies have nominally elucidated the prominence of such driving errors (Herslund \& Jörgensen, 2003; Rumar, 1990). For instance, Stutts et al. (2001) analyzed the National Accident Sampling System (NASS) Crashworthiness Data System (CDS) database from 1995 to 1999, using a set of driver attention categories at the time of the crash. Overall, $48.6 \%$ of drivers were classified as attentive when a crash occurred, $8.3 \%$ were distracted, and $5.4 \%$ of drivers 'looked but failed to see' a hazard prior to a crash. More females (6.2\%) than males (4.9\%) were involved in LBFTS crashes. When classifying using roadway factors, $88.3 \%$ of LBFTS crashes occurred at intersections and junctions. When environment and vehicle factors where analyzed, the highest frequency of LBFTS crashes occurred when more than one occupant was in the vehicle (33\%).

The purpose of this study was to examine the possibility of empirically producing the same pattern of LBFTS errors especially as the LBFTS phenomenon is affected by passenger conversation and gender. Crash analyses and prior research suggests that LBFTS crashes occur at intersections, involve attention-demanding situations, dual tasks, and may involve novice and older drivers to a greater extent (Caird et al., 2005; Famewo et al., in press; Kousanaï et al., 2008). LBFTS crashes occur when drivers are making turns or entering an intersecting roadway (Caird \& Hancock, 2007). Accordingly, dynamic change blindness (DCB) sequences were 
initiated as participants made a left turn maneuver in a driving simulator. Determination of the occurrence of LBFTS errors was made based on measures of eye movement fixations and responses to hazards.

Passengers are a well-known source of in-vehicle distraction (Stutts et al., 2001). Another objective was to determine whether a conversation with an attractive passenger, who is the opposite gender as the driver, affected drivers' detection of hazards in a left turn maneuver. A main effect of conversation condition was expected, where fewer hazards would be detected when drivers were engaged in a passenger conversation, compared to driving alone. The effects of social manipulations on driving performance have had minimal examination (Simons-Morton et al., 2005). Generally, conversation interaction differences can be categorized into visually oriented (men) and auditory oriented (women) (Orbuch \& Sprecher, 2003). Thus, driver performance was expected to differ according to gender differences, with males exhibiting increased glances off-road toward the passenger and females attending to a conversation more.

\section{METHODS}

\section{Participants}

Forty drivers (20 females, 20 males), between 18 and 25 years of age $(M=20.3)$ completed the one-hour study. An equal number of female (10) and male (10) drivers were assigned to each of the passenger and alone conditions. Sessions lasted about 1 hour and each student received course credit for his or her participation.

\section{Driving Simulator, Dynamic Change Blindness Events}

The University of Calgary Driving Simulator (UCDS) and ASL 501 eye tracker that was used in thus study is described in detail elsewhere (see Chisholm et al., 2008).

A dynamic change blindness (DCB) paradigm was developed that occluded the projected simulation scene with a black screen, returned to the traffic scene again, and alternated back and forth a number of times (Simons \& Rensink, 2005). Each sequence of alternating images started with a black screen for $100 \mathrm{~ms}$ followed by the reappearance of the traffic scene that contained an important hazard for $400 \mathrm{~ms}$. The black screen reappeared followed by the traffic scene again and so forth. The resulting visual effect appears as a 'flickering' of the screens. Seven alternations comprised one DCB sequence.

DCB sequences were initiated at intersections while participants were engaged in a left turn maneuver across dual oncoming lanes. Two independent roadway hazards were presented simultaneously; a motorcycle traveling at $65 \mathrm{~km} / \mathrm{h}$ straight through the intersection in the far lane and the pedestrian crossing the street (see Figures 1). The pedestrian walked across the corner and into the roadway inside of the crosswalk. During hazard-present DCB presentations, the pedestrian appeared and disappeared repeatedly. This scenario was designed to place the driver and pedestrian on a collision course with the participant. 


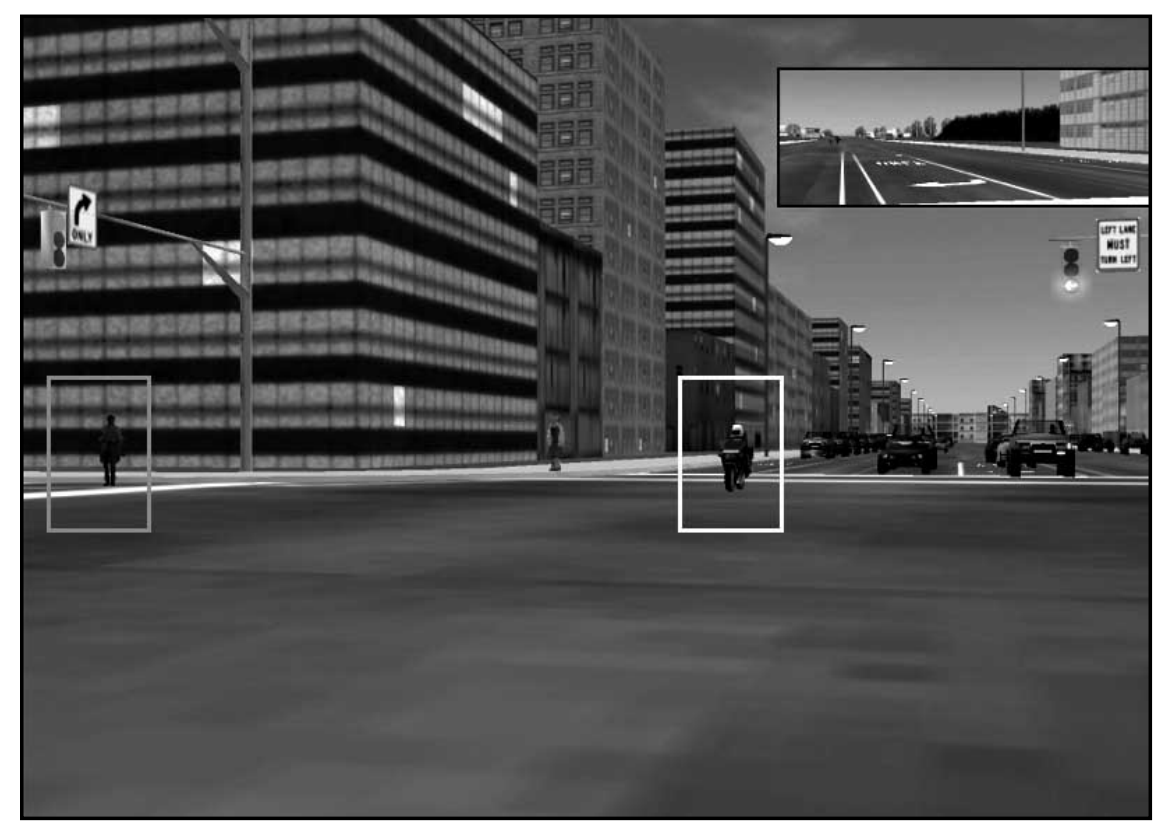

Figure 1. The positions of the motorcycle (right) and pedestrian (left) hazards

\section{Driving Scenarios and Procedures}

Each participant drove two simulated drives. The orientation drive also introduced participants to the DCB sequence. Throughout the experimental drive, participants were exposed to $12 \mathrm{DCB}$ sequences at intersections, nine of which were left turns and three were right turns. Six of the left turns were pedestrian-present DCB sequences, with the remaining DCB exposures containing no change at all.

The experimental drive was divided into two conditions, alone and with a passenger. If assigned to the passenger condition, a participant would converse with a confederate who was seated in the passenger seat. Two research assistants, a 24-year old female and a 19-year old male, served as passengers in the study. These research assistants were paired with participants of the opposite gender. The task of both research assistants was to be physically attractive, engage in selfdisclosure, maintain a pleasant affect, and express self-enhancing statements (i.e. verbal agreement, offer compliments) towards the driver. To accommodate gender specific preferences the male assistant insinuated future earning potential by mentioning a future lucrative career (a medical doctor), and was as jovial and humorous as possible. The female confederate wore formfit clothing with a low neckline and responded in a positive and affirmative manner to comments made by the driver. These criteria were expected to enhance drivers' willingness to divert attention from the road to the conversation, and achieve a cognitively distracting situation. 


\section{RESULTS}

\section{Experimental Design and Variables}

The 2 × 2 between-subjects experimental design had Gender (female, male) and Conversation (alone, passenger) as factors. Whether or not a driver looked at and/or responded to a hazard was coded to create a looked-but-failed-to-see dependent variable and was analyzed using discriminant function analysis or DFA. Additional analyses on collisions, near-misses, and social factors were performed but are not presented here.

\section{Looked-But-Failed-To-See (LBFTS) Errors}

Eye movements and hazard responses were used to classify participant responses into LBFTS categories. Two criteria were used; namely, "did the driver look at the hazard?" (yes or no) and "did the driver respond to the hazard?" (yes or no). Four possible cells result when these criteria are crossed (see Table 1). Eye movements were classified as a fixation, where three consecutive video frames (approximately $100 \mathrm{~ms}$, at $30 \mathrm{~Hz}$ ) were classified as a glance at the target. Thus, eye movements that landed within the area of the hazard (i.e. the size of the motorcycle or pedestrian) for this time (100 ms) were defined as target fixations and classified as looked. Drivers' actions were coded as having responded when he or she executed an appropriate evasive action (i.e., braking, accelerating, and/or steering) in time to avoid a collision or nearcrash.

Table 1. Classification of driver responses and eye-glance behavior to hazards

Response

(no, yes)

Eye-glance Behaviour (no, yes)

\begin{tabular}{|c|c|}
\hline $\begin{array}{c}\text { 1) Did not look at hazard, } \\
\text { (Failed to look, FTL) }\end{array}$ & $\begin{array}{c}\text { 2) Did not look at hazard, } \\
\text { Responded to hazard } \\
\text { (Responded, but did not look, } \\
\text { RDNL) }\end{array}$ \\
\hline $\begin{array}{c}\text { 3) Looked at hazard, } \\
\text { Did not respond to hazard } \\
\text { (Looked, but failed to see, } \\
\text { LBFTS) }\end{array}$ & $\begin{array}{c}\text { 4) Looked at hazard, } \\
\text { Responded to hazard } \\
\text { (Correct response, CR) }\end{array}$ \\
\hline
\end{tabular}

\section{LBFTS: Pedestrian Hazard}

The LBFTS category was of primary interest. Figure 2 shows the total number of pedestrian and motorcycle LBFTS incidents per condition. Women and men with passenger had higher motorcycle and pedestrian LBFTS incidents than when driving alone. 


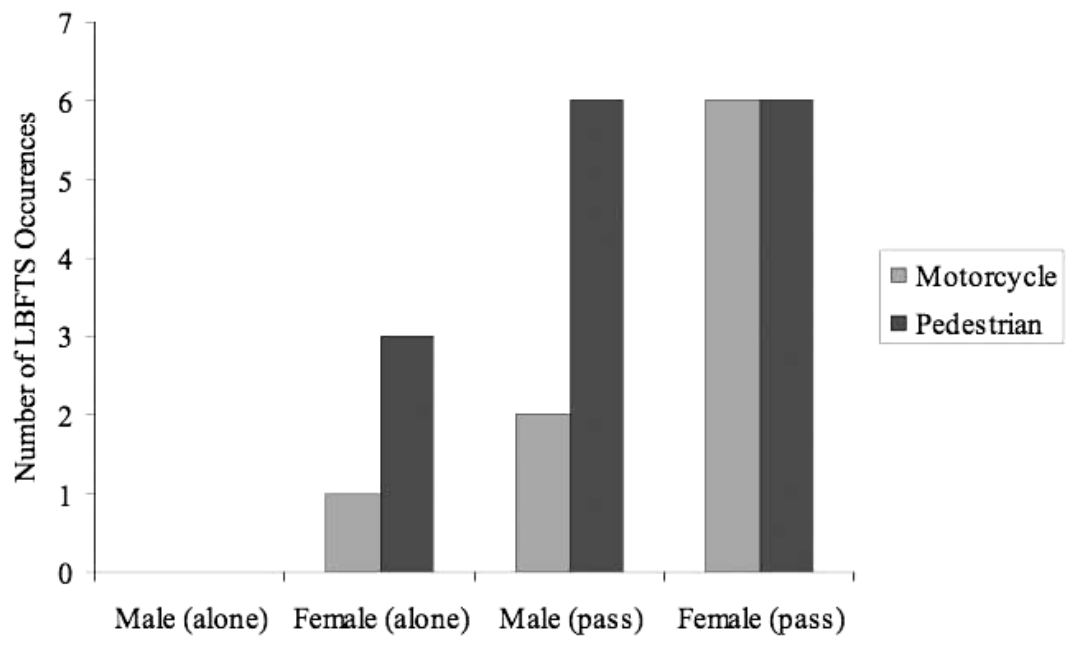

Figure 2. The occurrence of looked-but-failed-to-see errors by condition and hazard type

Passenger conversation (alone, passenger) and driver gender (male, female) were analyzed using a discriminant function analysis (DFA) in SPSS (v.13). DFA is used to predict group membership from a set of variables and indicate the accuracy of the predicted classification. Additionally, DFA examines to what degree the groups can be separated, or distinguished, from each other on the basis of the predictor variables (Tabachick \& Fidell, 2007). DFA can answer the question was whether the passenger's conversation or a driver's gender would predict LBFTS classification or group membership.

Two discriminate functions were calculated. Homogeneity of variance was not violated, as indicated by a non-significant Box's M statistic (11.597), $p=.281$. The first function was significant, demonstrating a strong association between classification groups and predictor variables, $\chi^{2}(6)=25.79 p<.0001$. Significant separation of one of the groups was observed $(\Lambda=$ $.86)$. The second function was not found to be significant, $\chi^{2}(2)=3.54, p=.171$, nor was the separation between groups as substantial $(\Lambda=.98)$. A difference in the amount of variance accounted for was also observed between each function, the first function accounted for $86.9 \%$ $(\lambda=.13)$ of the variance whereas the second function only explained $13.1 \%(\lambda=.02)$. Accuracy of group classification is indicated by the variance between scores on the function and the actual group they belong to $\left(\mathrm{R}_{\mathrm{c}}{ }^{2}\right)$. Function 1 demonstrated greater accuracy at predicting group membership, $\mathrm{R}_{\mathrm{c}}{ }^{2}=.34$, than Function $2, \mathrm{R}_{\mathrm{c}}{ }^{2}=.14$.

As indicated by group centroids, the mean discriminant scores for each group, Function 1 maximally separated LBFTS (.675) from no look/responded (-.862) and looked/responded (.706). A conventional rule of thumb, cut-off threshold of $+/-.30$, was used to indicate predictor relevance, given that there is no statistical test. Both variables loaded substantively, conversation (.84) and gender (.51), suggesting that these are efficient predictors for distinguishing when a driver will look but fail to see a hazard from when responding to both fixated and not fixated hazards. Predictor variable means provided evidence that drivers who looked but failed to see the pedestrian were also involved in a conversation $(M=1.80, S D=.41)$ and were also more likely 
to be female $(M=1.60, S D=.51)$, compared to looked/responded drivers; conversation $(M=$ $1.31, S D=.48)$ and gender $(M=1.15, S D=.38)$, and no look/responded drivers; conversation $(M=1.08, S D=.28)$ and gender $(M=1.39, S D=.51)$.

\section{DISCUSSION}

The purpose of this study was to examine the effects of a conversation on hazard detection and to determine if the looked-but-failed-to-see or LBFTS phenomenon could be elicited in an experimental setting. A combination of driver's eye movements and performance responses were used to determine LBFTS errors. The dynamic change blindness methods produced the lookedbut-failed-to-see phenomenon in the simulator. Drivers looked at hazards but did not make a proper response. The discriminant function analyses identified conversation and gender as reasonable predictors of pedestrian and motorcycle LBFTS errors. Eighty percent of pedestrian were correctly classified using conversation and gender as predictors. Conversation (.84) was a more powerful predictor relative to gender (.51) for the pedestrian hazard. Commission of LBFTS errors occurred primarily when engaged in a conversation, and slightly more often by female drivers than male drivers. The present results of this study indicate that distraction from a passenger conversation affects the depth of processing individual hazards.

In addition to conversation, results from the discriminant function analyses also indicated that gender was a predictor, though not as powerful as conversation, with female drivers being more likely to commit LBFTS errors than male drivers. The question then becomes "are women are more susceptible to attentional failure"? Not necessarily. The use of an in-vehicle conversation may have had differential effects on drivers, depending on gender. The design and use of a naturalistic conversation was specifically intended to be highly engaging to encourage participant interaction. Additionally, attraction, which is positively related to an individual's willingness to engage with a stranger, was optimized according to available gender preferences (Orbuch \& Sprecher, 2003). The majority of the interaction between driver and passenger occurred while seated in the simulator and the participant drove. In addition to the forwardfacing orientation, drivers wore restricting eye movement headgear in a dark laboratory. It is likely that these environmental factors limited drivers' head rotation and line-of-sight. Thus, the context of the simulator was not optimal for drivers to view their passengers. It is likely that these restrictions hindered male drivers' view of the female passenger, and consequently the degree to which they attended and/or engaged with the confederate. The attraction literature suggests the lack of visual access would not have affected female drivers' willingness to socially engage with the male passenger. This willingness to direct attention toward the passenger by females, and not males, may have resulted in greater cognitive distraction for female drivers, and thus higher LBFTS rates. 


\section{REFERENCES}

Caird, J. K., Edwards, C. J., Creaser, J. I., \& Horrey, W. J. (2005). Older drivers failures of attention at intersections using change blindness methods to assess turn decision accuracy. Human Factors, 47(2), 235-249.

Caird, J. K., \& Hancock, P. A. (2007). Left-turn and gap acceptance crashes. In R. E. Dewar \& P. L. Olson (Eds.), Human Factors in Traffic Safety ( $2^{\text {nd }}$ Ed., pp. 613-645). Tucson, AZ: Lawyers and Judges Publishing Company, Inc.

Chisholm, S.L., Caird, J.K., \& Lockhart, J. (2008). The effects of practice with MP3 players on driving performance. Accident Analysis and Prevention, 40, 704-713.

Famewo, J., Trick, L.M., \& Nonnecke, B. (in press). How does driving experience affect allocation of attention in complex signaled intersections? Proceedings of Vision in Vehicles XI, Dublin, Ireland.

Herslund, M.-B., \& Jörgensen, N. O. (2003). Looked-but-failed-to-see-errors in traffic. Accident Analysis \& Prevention, 35(6), 885.

Hills, H. L. (1980). Vision, visibility, and perception in driving. Perception, 9(2), 183-216.

Kousanaï, A., Boloix, E., van Elslande, P., \& Bastien, C. (2008). Statistical analysis of "looked but failed to see" accidents: Highlighting the involvement of two distinct mechanisms. Accident, Analysis and Prevention, 40, 461-469.

Orbuch, T. L., \& Sprecher, S. (2003). Attraction and interpersonal relationships. In J. Delamater (Ed.), Handbook of Social Psychology (pp. 339-362). New York, NY: Kluwer Academic/Plenum Publishers.

Rumar, K. (1990). The basic driver error: Late detection. Ergonomics, 33(10/11), 1281-1290.

Shaffer, D. R., \& Bazzini, D. G. (1997). What do you look for in a prospective date? Reexamining the preferences of men and women who differ in self-monitoring propensities. Personality and Social Psychology Bulletin, 23(6), 605-616.

Simons, D.J. \& Rensink, R.A. (2005). Change blindness: Past, present future. Trends in Cognitive Sciences, 9(1), 16-20.

Simons-Morton, B., Lerner, N., \& Singer, J. (2005). The observed effects of teenage passengers on the risky driving behavior of teenage drivers. Accident Analysis \& Prevention, 37(6), 973982.

Stutts, J., C., Reinfurt, D. W., Staplin, L., \& Rodgman, E. A. (2001). The role of driver distraction in traffic crashes. Washington, DC: AAA Foundation for Traffic Safety.

Tabachnick, B., \& Fidell, L. (2007). Using Multivariate Statistics (5th ed.). Needham Heights, MA: Allyn \& Bacon.

Treat, J. R. (1980). A study of precrash factors involved in traffic accidents. HSRI Research Review, 10(1), 1- 35. 